\title{
Analysis of Doubly Salient Memory Motors Using Preisach Theory
}

\author{
Yu Gong ${ }^{1,2}$, K. T. Chau ${ }^{1}$, J. Z. Jiang ${ }^{2}$, Chuang $\mathrm{Yu}^{1}$, and Wenlong $\mathrm{Li}^{1}$ \\ ${ }^{1}$ Department of Electrical and Electronic Engineering, The University of Hong Kong, Hong Kong, China \\ ${ }^{2}$ Department of Automation, Shanghai University, Shanghai, China
}

\begin{abstract}
With the introduction of doubly salient memory (DSM) motors, the flux controllability and hence the speed range of permanent magnet (PM) motors have reached a new height. This paper presents a new method to accurately analyze the DSM motor. The key is to incorporate the general expressions of the Preisach hysteresis model (PHM) of the AINiCo-PM into the time-stepping finite element method (TS-FEM). Based on the proposed PHM-TS-FEM, both static and transient performances of the DSM motor are successfully simulated. Finally, the validity and accuracy of the proposed method are verified by experimental results.
\end{abstract}

Index Terms-Memory motor, permanent magnet (PM), Preisach hysteresis, time-stepping finite element method (TS-FEM).

\section{INTRODUCTION}

$\mathbf{P}$ ERMANENT magnet (PM) motors are attractive for those applications desiring high efficiency and high power density [1]. Because of the inherent difficulty in flux control, the PM motors generally suffer from a limited speed range. Recently, two kinds of flux-controllable PM motors have been proposed: namely, the PM hybrid motors which utilize an additional dc field winding to strengthen or weaken the PM flux [2], [3] and the memory motors in which the AlNiCo-PM can be online magnetized or demagnetized with various magnetization levels [4], [5]. The memory motors take the definite advantage of higher efficiency than the PM hybrid motors, since the required current for PM magnetization is temporary whereas the required dc current for field hybridization is continual. Among two kinds of memory motors, namely the rotor-PM type [4] and the stator-PM type [5], the stator-PM memory motor utilizes the doubly salient structure to enable all PMs locating in the stator, so-called the doubly salient memory (DSM) motor, hence improving the mechanical robustness for high-speed operation.

Compared with other PM motors which are designed to guarantee the PMs always operating in the second quadrant of the B-H loop even under overload or transient fault conditions, the memory motors purposely operate over four quadrants to enable flux control. Thus, the available time-stepping finite element method (TS-FEM) [6] that was developed for conventional PM machines is unable to provide an accurate analysis for the memory motors. Very recently, a coupled Preisach modeling and FEM technique has been proposed to analyze the static performance including the air-gap flux density distributions and flux pattern of magnets in a rotor-PM memory motor [7]. However, the corresponding Preisach model involves the use of the Everett function, which needs many statistical experimental data. Therefore, there is a need to take an improved method aiming to provide more static and transient analysis results.

The purpose of this paper is to present a new method to accurately analyze both the static and transient performances of the DSM motor. The key is to incorporate the general expressions of the Preisach hysteresis model (PHM) [8], [9] into the

Manuscript received March 05, 2009. Current version published September 18, 2009 Corresponding author: Y. Gong (e-mail: yugong@eee.hku.hk).

Digital Object Identifier 10.1109/TMAG.2009.2021409
TS-FEM in such a way that the transient process of magnetization and demagnetization of the AlNiCo-PM can be performed. On top of using computer simulation to illustrate the validity of the proposed method, experimental verification will be given to verify its accuracy for transient analysis of the DSM motor.

\section{PRoposed Method}

\section{A. $P H M$}

Preisach theory describes the hysteresis of ferromagnetic materials as an infinite set of magnetic dipoles which have rectangular hysteresis loops. So, the PHM of the flux density $B$ induced by the field intensity $H$ can be expressed as [9]

$$
\begin{aligned}
B & =\iint_{S} \mu(\alpha, \beta) \gamma_{\alpha \beta}(H) d \alpha d \beta \\
& =\iint_{S^{+}(t)} \mu(\alpha, \beta) d \alpha d \beta-\iint_{S^{-}(t)} \mu(\alpha, \beta) d \alpha d \beta
\end{aligned}
$$

where $S^{+}(t)$ and $S^{-}(t)$ are the positive and negative sets of hysterons of the Preisach triangular region, $\mu(\alpha, \beta)$ is the Preisach distribution function of the dipoles, $\alpha$ and $\beta$ are the thresholds of the polar reversion of the dipoles with $H_{s} \geq \alpha \geq \beta \geq-H_{s}, H_{s}$ is the saturation magnetic field strength, and $\gamma_{\alpha \beta}$ is the switch function with $\gamma_{\alpha \beta}=1$ on $S^{+}$and $\gamma_{\alpha \beta}=-1$ on $S^{-}$. Consequently, the general expressions of the PHM can be written as

$$
\begin{aligned}
& \text { Initial curve (IC) }: B_{i}(H)=T(H,-H) \\
& \text { Download curve }: B(H)=B\left(H_{n}\right)-2 T\left(H_{n}, H\right) \\
& \text { Upward curve : } B(H)=B\left(H_{n}\right)+2 T\left(H, H_{n}\right)
\end{aligned}
$$

where the subscripts $i$ and $n$ denote the initial and the $n$th reversal point of the B-H curve, respectively; and $T(\alpha, \beta)$ is defined as

$$
T(\alpha, \beta)=\frac{B_{u}(\alpha)-B_{d}(\beta)}{2}+F(\alpha) F(-\beta)
$$

where the subscripts $u$ and $d$ refer to the upward limiting curve (ULC) and the downward limiting curve (DLC), respectively; and $F(\alpha)$ is given by

$$
F(\alpha)=\left\{\begin{array}{ll}
\frac{B_{d}(\alpha)-B_{u}(\alpha)}{2 \sqrt{B_{d}(\alpha)}} & \alpha \geq 0 \\
\sqrt{B_{d}(\alpha)} & \alpha \leq 0 .
\end{array} .\right.
$$




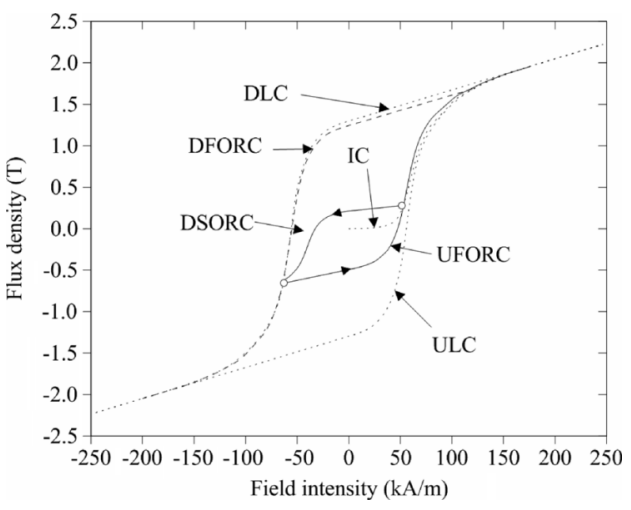

Fig. 1. Hysteresis curves of AlNiCo-PM.

Moreover, by substituting (5) and (6) into (2) and using the assumption that the limiting loop is symmetric about the origin, the IC can be expressed in terms of $B_{u}$ and $B_{d}$

$$
B_{i}(H)=\frac{\left[B_{u}(H)+B_{d}(H)\right]^{2}}{4 B_{d}(H)} .
$$

Fig. 1 shows the hysteresis curve of the AlNiCo-PM, depicting the IC, DLC, ULC, downward first-order reverse curve (DFORC), download second-order reverse curve (DSORC), and upward first-order reserve curve (UFORC).

It should be noted that the above general expressions of the PHM enables the generation of B-H trajectories, including the minor loops, based only on the input data of the limiting loop, without involving any statistically distributed parameters or empirical adjustment of the trajectories.

\section{B. Coupled PHM-TS-FEM}

In general, the field equation of all PM machines can be expressed as

$$
\nabla \times(\nu \nabla \times A)=J+\nabla \times\left(\nu B_{r}\right)-\sigma \frac{\partial A}{\partial t}
$$

where $A$ is the magnetic vector potential, $J$ stands for the current density in the armature and field slots, $B_{r}$ represents the residual flux density, and $\nu$ refers to reluctivity. The conventional TS-FEM simply treats the PM having linear $B_{r}$ and constant $\nu$. For the DSM motor, the AlNiCo-PM no longer exhibits linear $B_{r}$ and constant $\nu$. The magnetization or demagnetization curves are multivalued, depending on the history of the external magnetic field. In order to incorporate the above PHM of the AlNiCo-PM into the TS-FEM, a new algorithm is developed as depicted in Figs. 2 and 3.

Fig. 2 illustrates the flowchart to initialize the PHM of the AlNiCo-PM. Initially, each PM element is set to have a constant $\nu$ and zero $B_{r}$. Then, the FEM is applied to calculate the initial field intensity $H_{o}$ of each PM element by

$$
H_{0}=\nu\left(\nabla \times A-B_{r}\right)=\nu \nabla \times A .
$$

Hence, the initial flux density $B_{o}$ on the IC can be deduced from (7). After removing the initial external field, the reversal point will be determined according to the magnetization level. Namely, $B_{r}$ will be determined by the DLC if full magnetization has been achieved; otherwise, it will be determined by the DSORC if partial magnetization.

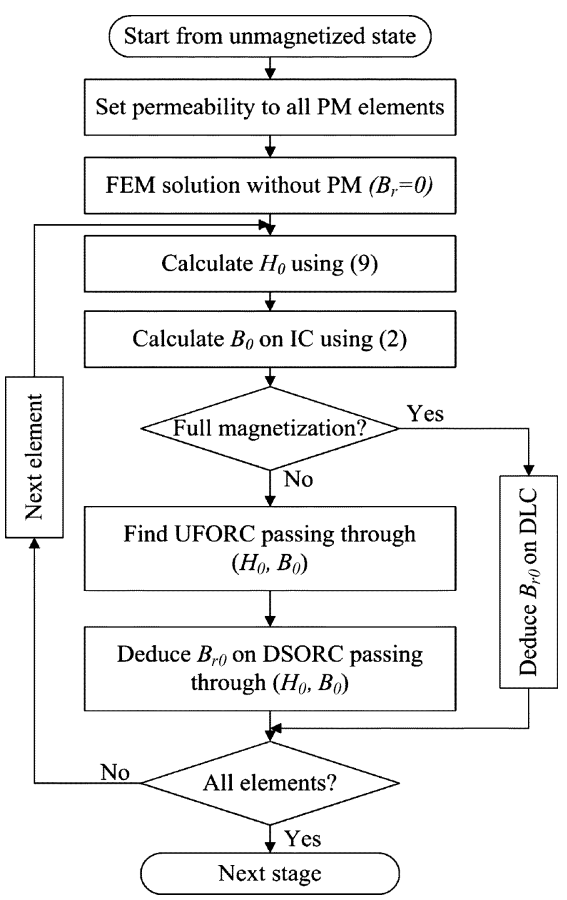

Fig. 2. Flowchart of initialization of AlNiCo-PM.

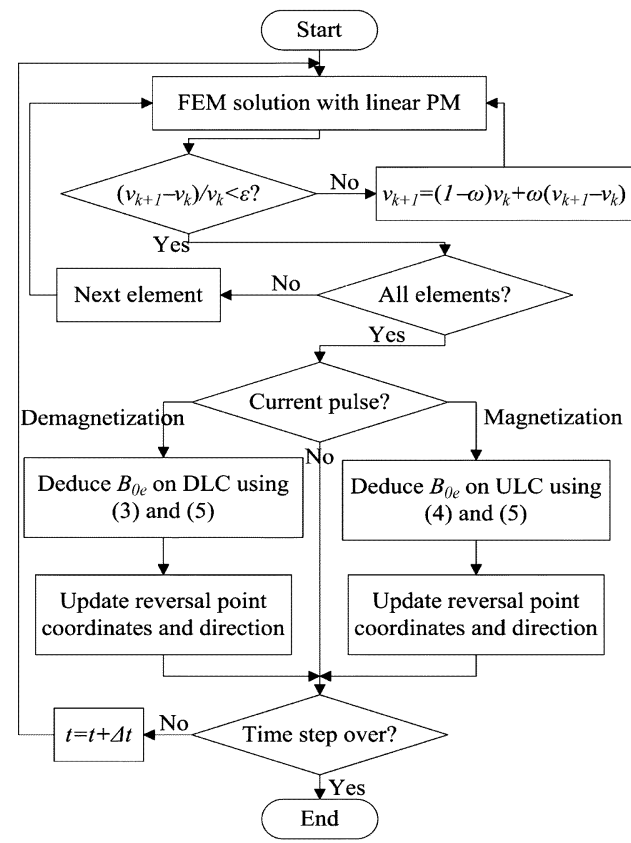

Fig. 3. Flowchart of adjustment in AlNiCo-PM.

Fig. 3 shows the flowchart to adjust the working point of each element in the AlNiCo-PM. From the previously determined $B_{r}$, the corresponding $\nu$ can be calculated by

$$
\nu=\frac{\partial H}{\partial B}=\frac{H}{B(H)-B_{r}} .
$$

The iteration process of each PM element will be governed by

$$
\begin{aligned}
\nu_{k+1} & =(1-\omega) \nu_{k}+\omega\left(\nu_{k+1}-\nu_{k}\right) \\
\frac{\nu_{k+1}-\nu_{k}}{\nu_{k}} & <\varepsilon
\end{aligned}
$$




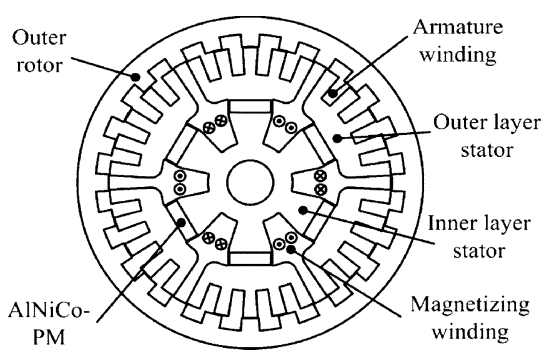

Fig. 4. Configuration of DSM motor.

TABLE I

Key Design Data

\begin{tabular}{|c|c|}
\hline Outer rotor diameter & $270 \mathrm{~mm}$ \\
\hline Air-gap length & $0.6 \mathrm{~mm}$ \\
\hline Number of armature winding / slot & $60 \mathrm{turns}$ \\
\hline Number of magnetizing winding / slot & 500 turns \\
\hline Stack length & $80 \mathrm{~mm}$ \\
\hline PM material & $\mathrm{AlNiCo}$ \\
\hline PM size (length $\times$ width) & $10 \times 30 \mathrm{~mm}^{2}$ \\
\hline PM maximum residual flux density & $1.3 \mathrm{~T}$ \\
\hline PM coercive force & $56 \mathrm{kA} / \mathrm{m}$ \\
\hline PM recoil permeability & $2.4 \sim 3.6$ \\
\hline
\end{tabular}

where $\omega$ is the iteration coefficient and $\varepsilon$ is the tolerance. After the convergence of $\nu$ for all PM elements, the hysteresis equations (3), (4), and (5) will be used to calculate $B_{0}$ from the corresponding curves, hence updating the coordinates and direction of the reversal points for next time step.

\section{DSM MOTOR}

Fig. 4 shows the configuration of the DSM motor [5]. It adopts a five-phase outer-rotor doubly salient PM topology. The rotor is made of solid iron with neither PMs nor windings, thus offering excellent mechanical robustness. The ac armature winding and dc magnetizing winding are located in the outer layer stator and inner layer stator, respectively. This arrangement can effectively utilize the space of the stator yoke. Also, it enables the PMs to be immune from accidental demagnetization by the armature field, which is important for the AlNiCo-PM having a low coercive force. Different from the conventional rotor-PM memory motor, which suffers from complexity for magnetization or demagnetization, this DSM motor can easily magnetize or demagnetize the AlNiCo-PM by using temporary current pulses.

\section{ANALYSIS AND EXPERIMENTAL RESUltS}

Both simulation and experimentation are used to illustrate the validity and accuracy of the proposed method. The key design data of the DSM motor are listed in Table I. It is also prototyped as shown in Fig. 5.

First, the radial air-gap flux density distributions under different magnetizing currents are shown in Fig. 6, in which the process is divided into the magnetizing state and the magnetized state. It can be seen that the flux density during the magnetizing state is higher than that during the magnetized state, which is due to the contribution from the dc magnetizing current and the recoil reduction of PM flux density after magnetization. It can also

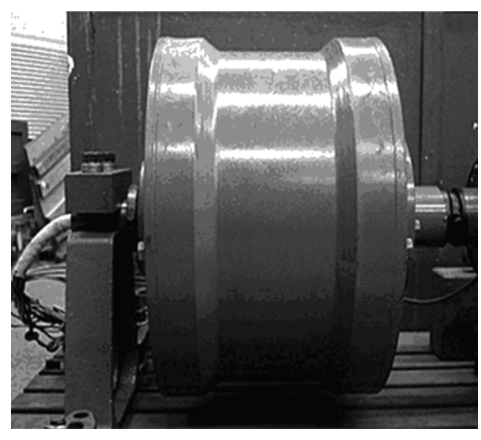

Fig. 5. Prototype of DSM motor.

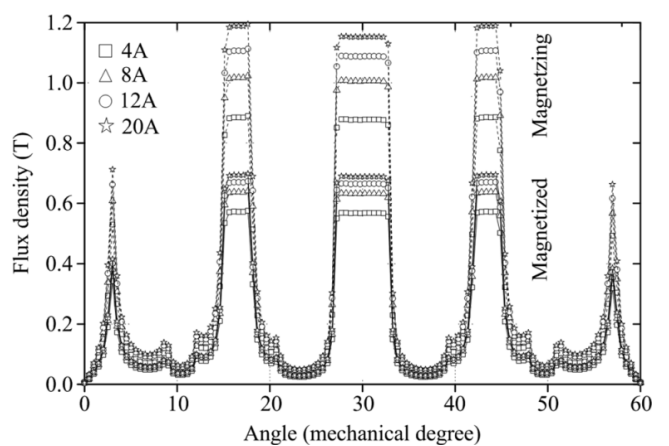

Fig. 6. Air-gap flux density distributions under various magnetizing currents.

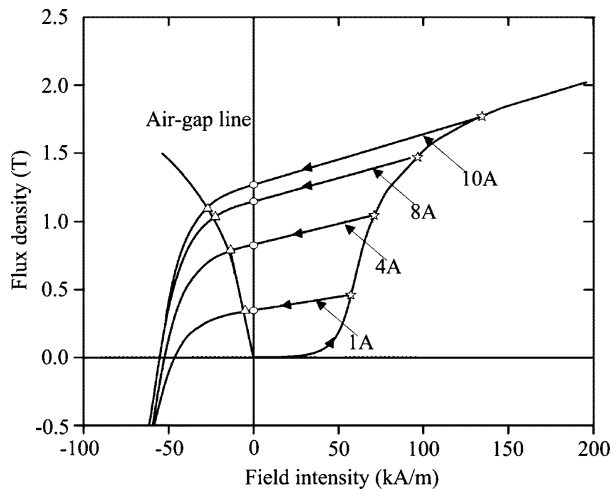

Fig. 7. Trajectories of AlNiCo-PM under various magnetizing currents.

be observed that the effect of increasing the magnetizing current magnitude is diminishing because of magnetic saturation.

Second, the trajectories of the AlNiCo-PM after applying various magnetizing currents are depicted in Fig. 7, in which the points with triangular marker are the corresponding operating points. Fig. 8(a) shows the simulated no-load EMF transient response at the speed of $100 \mathrm{rpm}$ when a magnetizing current pulse of $4 \mathrm{~A}$ is applied. On the other hand, the corresponding response excited by the same magnetizing current is measured as shown in Fig. 8(b). It can be found that these transient responses are closely matched, hence verifying the validity and accuracy of the proposed PHM-TS-FEM method for the DSM motor under online magnetization.

Third, the trajectories of the AlNiCo-PM after applying various demagnetizing currents are depicted in Fig. 9, in which the initial operating point is resulted from using the magnetizing current of $8 \mathrm{~A}$. It can be observed that the demagnetization is much easier than the magnetization. Also, Fig. 10 shows that 


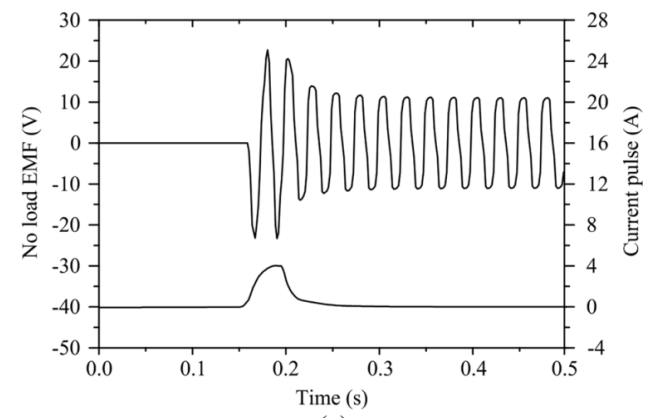

(a)

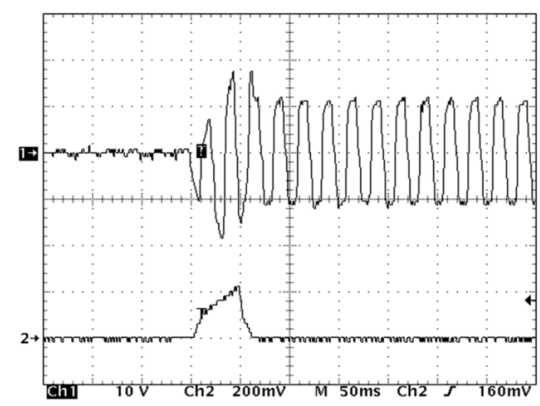

(b)

Fig. 8. Transient EMF responses under magnetizing current of $4 \mathrm{~A}$ at $100 \mathrm{rpm}$. (a) Simulated. (b) Measured (10 V/div, 4 A/div).

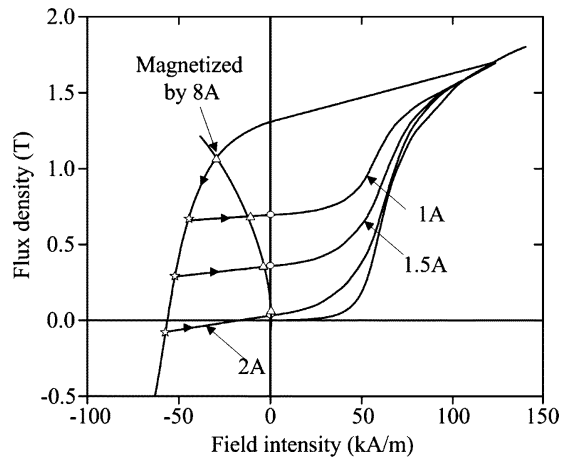

Fig. 9. Trajectories of AlNiCo-PM under various demagnetizing currents.

the simulated and measured no-load EMF transient responses at the speed of $100 \mathrm{rpm}$ under a demagnetizing current pulse of 1.5 A have good agreement, thus verifying the validity and accuracy of the proposed method for the DSM motor under online demagnetization.

\section{CONCLUSION}

In this paper, a new PHM-TS-FEM has been proposed and implemented for DSM motors. The key is to employ the general expressions of the PHM to model the AlNiCo-PM so that the generation of B-H trajectories can be simply based on the input data of the limiting loop, while avoiding any statistically distributed parameters or empirical adjustment of the trajectories. The resulting PHM-TS-FEM has been successfully applied to simulate both static and transient performances of the DSM motor. Particularly, the transient EMF responses under sudden applications of magnetizing and demagnetizing currents are simulated, and experimentally verified.

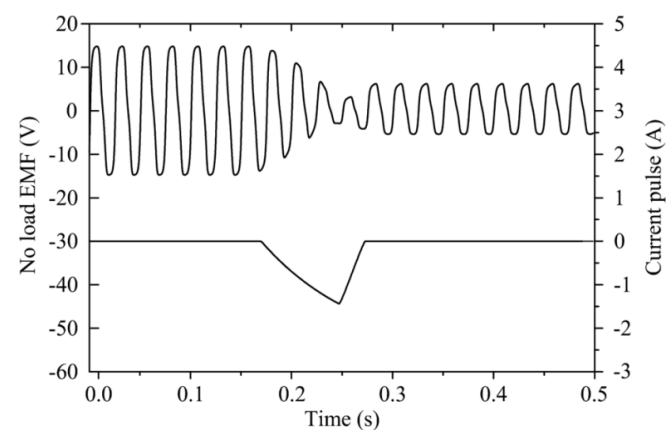

(a)

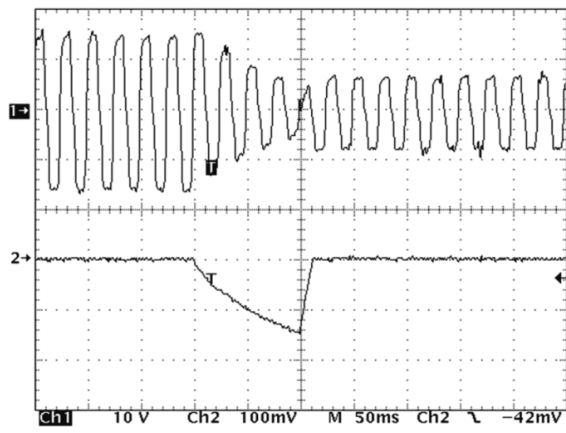

(b)

Fig. 10. Transient EMF responses under demagnetizing current of $1.5 \mathrm{~A}$ at 100 rpm. (a) Simulated. (b) Measured (10 V/div, 1 A/div).

\section{ACKNOWLEDGMENT}

This work was supported by a grant (HKU 7105/07E) from the Research Grants Council, Hong Kong Special Administrative Region, China.

\section{REFERENCES}

[1] K. T. Chau, C. C. Chan, and C. Liu, "Overview of permanent-magnet brushless drives for electric and hybrid electric vehicles," IEEE Trans. Ind. Electron., vol. 55, no. 6, pp. 2246-2257, Jun. 2008.

[2] K. T. Chau, Y. B. Li, and J. Z. Jiang, "Design and analysis of a stator doubly fed doubly salient permanent magnet machine for automotive engines," IEEE Trans. Magn., vol. 42, no. 10, pp. 3470-3472, Oct. 2006.

[3] C. Liu, K. T. Chau, J. Z. Jiang, and S. Niu, "Comparison of statorpermanent-magnet brushless machines," IEEE Trans. Magn., vol. 44, no. 11, pp. 4405-4408, Nov. 2008.

[4] V. Ostovic, "Memory motor-A new class of controllable flux PM machines for a true wide speed operation," IEEE Ind. Appl. Mag., vol. 9, no. 1, pp. 52-61, Jan.-Feb. 2003.

[5] C. Yu, K. T. Chau, X. Liu, and J. Z. Jiang, "A flux-mnemonic permanent magnet brushless motor for electric vehicles," J. Appl. Phys., vol. 103, no. 7, pp. 07F103:1-07F103:3, Apr. 2008.

[6] Y. Wang, K. T. Chau, C. C. Chan, and J. Z. Jiang, "Transient analysis of a new outer-rotor permanent-magnet brushless dc drive using circuitfield-torque time-stepping finite element method," IEEE Trans. Magn., vol. 38, no. 2, pp. 1297-1300, Mar. 2002.

[7] J. H. Lee and J. P. Hong, "Permanent magnet demagnetization characteristic analysis of a variable flux memory motor using coupled Preisach modeling and FEM," IEEE Trans. Magn., vol. 44, no. 6, pp. $1550-1553$, Jun. 2008.

[8] S. R. Naidu, "Simulation of the hysteresis phenomenon using Preisach's theory," IEE Proc. A, vol. 137, no. 2, pp. 73-79, Mar. 1990.

[9] S. Y. R. Hui and J. G. Zhu, "Numerical modeling and simulation of hysteresis effect in magnetic cores using transmission-line modeling and the Preisach theory," IEE Proc.-Electric Power Appl., vol. 142, no. 1, pp. 57-62, Jan. 1995. 